\title{
DO-IT-YOURSELF ANALYSIS OF PRECISION ELECTROWEAK DATA
}

\author{
V.A. Novikov \\ ITEP, Moscow, 117259, Russia \\ L.B. Okun \\ ITEP, Moscow, 117259, Russia \\ and \\ Theoretical Physics Division, CERN \\ CH-1211 Geneva 23 \\ and \\ M.I. Vysotsky \\ ITEP, Moscow, 117259, Russia

\section{SECOND ERRATUM}

In the text, including Eqs. (8) and (9) and figure captions, $300 \mathrm{GeV}$ should be changed to $200 \mathrm{GeV}$ for $m_{H}$, and $135 \mathrm{GeV}$ to $140 \mathrm{GeV}$ for $m_{t}$.

On page 1, the last sentence of the second paragraph from the bottom (the fifth from the top) should be deleted.

CERN-TH.6715/92/ERRATUM/2

February 1993 